\title{
Multi Drug Resistant Bacteria: Prevalence and Associated Risk Factors Amongst ICU Health Care Workers of a Tertiary Care Hospital
}

\author{
Iva Chandola ${ }^{1 *}$, Anurag Bijalwan², Nidhi Negi', Vijay Kataria ${ }^{1}$ \\ ${ }^{1}$ Dept of Microbiology, Shri Guru Ram Rai Institute of Medical and Health Sciences, Dehradun, India \\ ${ }^{2}$ Dept of Surgery, Shri Guru Ram Rai Institute of Medical and Health Sciences, Dehradun, India
}

\section{ABSTRACT}

Background: Multi Drug Resistant bacteria pose serious threat to patient safety worldwide. Health Care Workers are an important source of dissemination and transmission of these organisms to patients especially in intensive care units (ICU).Although many studies have been carried out in India which have determined the prevalence of either gram positive or gram negative MDR bacteria colonizing the HCW separately, no study so far has determined the prevalence and risk factors for acquisition of MRSA, VRE, MDR Acinetobacter baumanii \& Pseudomonas aeruginosa simultaneously in the same HCW population

Methods: Hand swabs from $198 \mathrm{HCW}$ were obtained, processed and isolates identified by automated method using Vitek II (Biomerieux, Durham, NC).Risk factor assessment was done based on a questionnaire using Fischer's exact /Chi square test.

Result: A total of $24 \mathrm{HCWs}$ (12.1\%) were found positive for MDR bacteria. At $5.1 \%(\mathrm{n}=10)$ MDR Acinetobacter baumanii was the most common isolate obtained. Majority of MDR bacteria (16.1\%) were isolated from hands of doctors. Male sex, presence of chronic /open wound and close contact with patients were factors found significantly associated with colonization of hands of $\mathrm{HCW}$.

Conclusion: Health Care Associated Infections in the vulnerable ICU patient population can be linked to the MDR bacterial flora of the HCWs. A.baumanii has been found to be most frequently contaminating the hands of HCW. Compliance with contact precautions, proper hand hygiene and adequate environmental cleaning may decrease this transmission.

\section{Keywords: Health Care Worker, Multi drug resistant Bacteria, hand hygiene, bacterial colonization}

\section{Introduction}

Multi drug resistant (MDR) bacteria pose serious threat to patient safety worldwide adversely affecting their mortality and morbidity despite antimicrobial therapy and advances in supportive care ${ }^{[1,2]}$ This is especially true for patients admitted in Intensive Care Units (ICU) who are more likely to be immunocompromised and suffering from serious underlying diseases ${ }^{[3,4]}$. Health care workers (HCW) are an important source of dissemination and transmission of these multidrug resistant organisms to patients ${ }^{[5]}$.

Although many studies, conducted in India and globally, have determined the prevalence of bacterial colonization and risk factors for their acquisition by HCWs including contact with wound dressing, linen, artificial airways, infusion pumps, catheters or drains or performing physical examination among others. Very few studies and to the best of our knowledge none so far in India, have accessed risk factors for contamination with the most common MDR bacteria(MRSA,ESBL,VRE, MDR non fermenters etc) simultaneously and on the same HCW population.
Globally studies have shown that 10 to $70 \%$ of nosocomial infections are preventable depending on setting, study design, baseline infection rate and type of infection ${ }^{[1]}$ Understanding the factors that lead to contamination of HCW hands in a particular setting is likely to help develop strategies unique to each institution to prevent transmission of MDR bacteria from $\mathrm{HCW}$ to patients and vice versa. Also it would help curb the menace of prescribing inadequate empirical antibiotic therapy to patients leading to increased morbidity and mortality, development of antimicrobial resistance and leading to unnecessary hospitalization.

The study was therefore carried out with the objective of determining the prevalence of MDR bacteria in the hand of ICU health care workers and the risk factors associated with the acquisition of these pathogens.

\section{Materials and Methods}

Study Design and Population: A prospective study was conducted at the 1000 bedded Shri Mahant Indresh Hospital (Dehradun, Uttrakhand) between June 2016 and December 2016. All samples were collected from the Medical ICU ( 26 bed unit), Surgical ICU ( 21 bed unit), Cardiac ICU (14 
bed Unit) and Pediatric ICU (28 bed unit). The study was approved by the Institutional Ethics committee.

Sample Collection: HCWs (nurses, technicians and doctors) were approached for participation in the study before engaging in routine, clinical care activities for patients. Samples were taken at the beginning of the day to determine the contamination that the HCWs were carrying with them and not which they may acquire during that particular days activity. Sterile rayon-tipped applicators were moistened with sterile normal saline and hand samples were obtained with a standardized process by swabbing the dorsum of each finger three times and the palm of each hand two times with a twirling motion of the swab with a single swab for both hands ${ }^{[1]}$.

Data Collection: Potential risk factors for MDR bacteria colonization were identified using a questionnaire. This included demographic data such as their age, sex, time for which they have been working in ICUs since prolonged exposure to ICU patients makes them more likely to acquire bacterial contamination), close contact with patient either in form of changing dressing, assisting mobility or carrying out an invasive procedure, presence of open/chronic wounds on exposed areas of the body in the last six months, chronic disorders, chronic skin condition( which are indicative of an immunocompromised state), contact with animals since house hold pets are a known source of infection of MDR bacteria of zoonotic origin such as MRSA, and Extended Spectrum Beta Lactamases producing E.coli ${ }^{[6]}$, their own hospital stay which is indicative of a illness severe enough to have compromised their immunity and making them an easier target for bacterial contamination and infection and use of antibiotics( usage of antibiotics especially the broad spectrum ones can favor colonization with MDR bacteria). The staff was asked to complete this questionnaire on their own.

Microbiological Processing: The moistened swabs were then transported to the Microbiology laboratory as soon as possible, dipped in sterile normal saline . Here, each sample was inoculated within an hour of collection in brain heart infusion broth and incubated for 24 hours at $37^{\circ} \mathrm{c}$. After incubation the broth was sub-cultured on 5\% sheep blood agar and Mac Conkey agar. All isolates were then identified and their antibiotic resistance profile determined by automated method using Vitek II (Biomerieux, Durham, NC) based on Clinical Laboratories and Standards Institute Guidelines (2016). Further, statistical analysis of data and risk factorestimation was done for isolates positive for MRSA, VRE, MDR Acinetobacter baumanii, MDR Pseusdomonas aeruginosa. MRSA and VRE were defined as per CLSI 2016 guidelines ${ }^{[7]}$. There are various definitions available in literature. for MDR Acinetobacter baumanii and MDR Pseusdomonas aeruginosat For this study, we have defined MDR Acinetobacter baumanii and MDR Pseusdomonas aeruginosa as, non susceptibility to at least one agent in three or more antimicrobial categories ${ }^{[8]}$.

Statistical Analysis: Risk factor analysis was conducted using the Fisher exact test to measure the significance of associations between binary variables and the dependent variable of MDR contamination of hands. We report adjusted odds ratios (aORs) and 95\% confidence intervals (CIs) from the multivariate logistic regression model. All statistical tests were 2 -sided; $\mathrm{P}<0.05$ was considered to be statistically significant. All analyses were performed using statistical software SPSS, version 22

\section{Result}

A total of 198 HCWs (140 females, 58 males) participated in the study. The health care staff included was between 21-65 years of age, with median age of 36. About half of them had worked in this field for at least 1 year while $21 \%$ had worked for more than 15 years. [Table 1]

Almost two thirds $(72.7 \%$ )of them mentioned that as part of their professional duties they had close contact with patients requiring care such as facilitating mobility, changing bandages or treatment of bed sores while close to half $(52 \%)$ reported to have close contact with animals. A total of $34.3 \% \mathrm{HCW}$ gave history of use of antibiotics in last six months while $11.1 \%$ were admitted in hospital for varied ailments in last one year. Chronic skin disease and chronic wounds were present in $3.6 \% \mathrm{HCW}$ respectively while $1.6 \%$ were found suffering from diabetes mellitus.

Mdr Bacteria Prevalence: Hand swabs were taken from 123 nurses, 44 patient care technicians and 31 doctors. Bacterial isolates were recovered from hand swabs of 148(74.75\%) HCWs. These included both commensals as well as known pathogens. A total of 56(28.28\%) $\mathrm{HCW}$ showed growth of more than one bacteria. Of these $133(68.18 \%)$ were established pathogens namely Acinetobacter spp 36.48\%, Pseudomonas spp 12.19\%, Klebsiella spp 7.31\%, S.aureus 7.31\%, Enterococcus spp $4.89 \%$ while rest 15 were commensals (Table 1 ).

A total of $24 \mathrm{HCWs}(12.1 \%)$ were found to be carrying MDR bacteria on their hands distribution of which is as follows: MDR Acinetobacter baumanii 10(5.1\%), MDR Pseudomonas aeruginosa 7(3.4\%) MRSA were 5(3.2\%), and Vancomycin Resistant Enterococcus 2(0.6\%) (Table 2).

Majority of MDR ( $n=13 / 24,54.16 \%)$ were isolated from hands of doctors followed by technicians $(n=6 / 24,25 \%)$ and least number was recovered from hands of nurses $(n=5 / 24$, 
20\%) (Chart 1).The most commonly isolated MDR from both doctors and nurses was Acinetobacter baumanii ( $\mathrm{n}=7$ and 3 respectively) while it was Pseudomonas aeruginosa $(\mathrm{n}=3)$ from Technicians (Fig1).

Risk factors significant for MDR bacterial contamination of HCWs hands were evaluated with bivariate analysis (Table 3) followed by multiple logistic regression analysis

The number of males affected was significantly higher than females ( $50 \%$ vs $2.5 \%$ respectively; $\mathrm{p}<0.05$; Table 3).No difference in susceptibility to MDR bacteria was found in terms of age, occupation and time spent in health care $(\mathrm{p}>0.05)$. Time spent in ICU health care (aOR 4.856;95\% CI 0.495-47.652), Occpation(aOR 1.912; 95\%CI 0.959-3.812), Contact with animals (aOR 4.663; 95\%CI $0.289-75.329$ ), Use of antibiotics in last six months (aOR 4.856;95\%CI 0.495-47.652), Hospital admission in last 1 year(aOR 2.610;95\%CI 1.339 - 5.088), Chronic skin disease (aOR 0.905; 95\% CI 0.905 - 5.654), Dibetes mellitus (aOR 9.938; 95\% CI 1.617-61.069), were factors not found to be significantly associated with colonization of hands of HCW with MDR bacteria ( $\mathrm{p}>0.05)$

However close contact with ICU patients (aOR 1.125; 95\% CI 0.475- 2.667) and having acquired askin ulcer / wound in last six months (aOR 0.677; 95\% CI 0.383-1.196) was found to be significantly associated with carriage of MDR bacteria by HCW $(\mathrm{p}<0.05)$.

\section{Discussion}

The study on MDR bacteria in ICU health workers marks the first time such data has been made available for the

Table 1: Organism isolated from hand swabs of HCW

\begin{tabular}{|c|c|c|}
\hline ORGANISM ISOLATED & NUMBER & PERCENTAGE \\
\hline Staphylococcus aureus & 14 & 7.31 \\
\hline Klebsiella spp & 14 & 7.31 \\
\hline Enterococcus spp & 9 & 4.89 \\
\hline Acinetobacter spp & 72 & 36.48 \\
\hline Pseudomonas spp & 24 & 12.19 \\
\hline Total & 133 & 68.18 \\
\hline
\end{tabular}

Table 2: Prevalence of MDR bacteria amongst HCW

\begin{tabular}{|c|c|c|}
\hline MDR & Number & Percentage \\
\hline VRE & 2 & 0.6 \\
\hline MRSA & 5 & 3.2 \\
\hline MDR Pseudomonas aeruginosa & 7 & 3.4 \\
\hline MDR Acinetobacter baumanii & 10 & 5.1 \\
\hline Total & 24 & 12.3 \\
\hline
\end{tabular}

Annals of Pathology and Laboratory Medicine, Vol. 4, Issue 5, September-October, 2017 state of Uttrakhand and one of the very few studies in India which have determined the risk factors for colonization of hands of $\mathrm{HCW}$ with four types of MDR bacteria simultaneously in the same population. The risk factors found to be statistically associated with MDR bacteria colonization include male sex, chronic skin disease and close contact with ICU patient. separately determined the prevalence of MRSA 1-3\%[9,10], Acinetobacter baumanii 5\% ${ }^{[11]}$ Pseudomonas aeruginosa $2.5 \%$ (11) but very few have determined the prevalence of all four MDR bacteria (MRSA, VRE, MDR Pseudomonas aeruginosa, MDR Acinetobacter baumanii) simultaneously on the same health care population along with their associated risk factors. The prevalence rates in these studies are however similar to those found in our study: Acinetobacter baumanii 5.1\%, Pseudomonas aeruginosa $3.4 \%$, MRSA $3.2 \%$, VRE(0.6\%).

We have found that hands of HCW were more frequently contaminated with MDR Acinetobacter baumanii(5.1\%) than with other MDR bacteria. MDR Acinetobacter baumanii has also been detected with higher frequency as compared to other MDR bacteria in a study by Morgan et $\mathrm{a}^{[1]}$.This relatively high frequency suggests that $A$. baumannii has a higher propensity to be transmitted to HCWs than do other MDR bacteria. This may be a factor in nosocomial spread, explaining in part the recent worldwide emergence of MDR A. baumannii ${ }^{[13]}$

Also hands of doctors were more frequently contaminated $(16.1 \%)$ than those of technicians $(13.6 \%)$ and nurses
Many studies are available from India which have 
Table 3: Description of study population and MDR positive cases among Health Care workers.

\begin{tabular}{|c|c|c|c|c|c|c|}
\hline \multicolumn{2}{|l|}{ Variable } & \multirow{2}{*}{$\begin{array}{l}\text { HCW total } \\
\mathbf{N}=198 \\
158(79.8)\end{array}$} & \multirow{2}{*}{$\begin{array}{c}\text { MDRO staff } \\
\text { N = } 24 \# \\
4(2.5)\end{array}$} & \multirow[t]{2}{*}{ p value \#\# } & \multirow[t]{2}{*}{ aOR } & \multirow[t]{2}{*}{$95 \% \mathrm{Cl}$} \\
\hline & female & & & & & \\
\hline Sex & male & $40(20.2)$ & $20(50)$ & 0.02 & & \\
\hline \multirow{5}{*}{ Age in years } & $<30$ & $43(21.7)$ & $9(20.9)$ & & & \\
\hline & $30-39$ & $42(21.2)$ & $4(9.5)$ & & & \\
\hline & $40-49$ & $47(23.7)$ & $3(6.4)$ & & & \\
\hline & $50-59$ & $52(26.3)$ & $5(9.6)$ & & & \\
\hline & $>60$ & $14(7.1)$ & $3(21.4)$ & 0.25 & 4.228 & $2.058-8.686$ \\
\hline \multirow{5}{*}{ Time spent in ICU health care } & $<1 \mathrm{yr}$ & $30(15.1)$ & $3(10)$ & & & \\
\hline & $1-5 y r$ & $58(29.3)$ & $5(8.6)$ & & & \\
\hline & $6-10 \mathrm{yr}$ & $38(19.2)$ & $4(10.5)$ & & & \\
\hline & $11-15 \mathrm{yr}$ & $31(15.6)$ & $2(6.4)$ & & & \\
\hline & $>15 \mathrm{yr}$ & $41(20.7)$ & $1(2.4)$ & 0.96 & 4.856 & $0.495-47.652$ \\
\hline \multirow{3}{*}{ Occupation } & Nurse & $123(62.1)$ & $13(10.6)$ & & & \\
\hline & Technician & $44(22.2)$ & $6(13.6)$ & & & \\
\hline & Doctor & $31(15.6)$ & $5(16.1)$ & 0.47 & 1.912 & $0.959-3.812$ \\
\hline Close contact with patients & & $144(72.7)$ & $12(8.3)$ & 0.02 & 1.125 & $0.475-2.667$ \\
\hline Contact with animals & & $103(52)$ & $2(1.9)$ & 1 & 4.663 & $0.289-75.329$ \\
\hline Use of antibiotics last six months & & $68(34.3)$ & $2(2.9)$ & 0.18 & 4.856 & $0.495-47.652$ \\
\hline Hospital admission last one year & & $22(11.1)$ & $7(31.8)$ & 0.17 & 2.610 & $1.339-5.088$ \\
\hline Chronic skin disease & & $7(3.5)$ & $1(14.2)$ & 0.06 & 2.263 & $0.905-5.654$ \\
\hline Diabetes mellitus & & $21(10.6)$ & $1(4.7)$ & 1 & 9.938 & $1.617-61.069$ \\
\hline Wound or ulcer in last six months & & $7(3.6)$ & $2(20)$ & 0.04 & 0.677 & $0.383-1.196$ \\
\hline
\end{tabular}

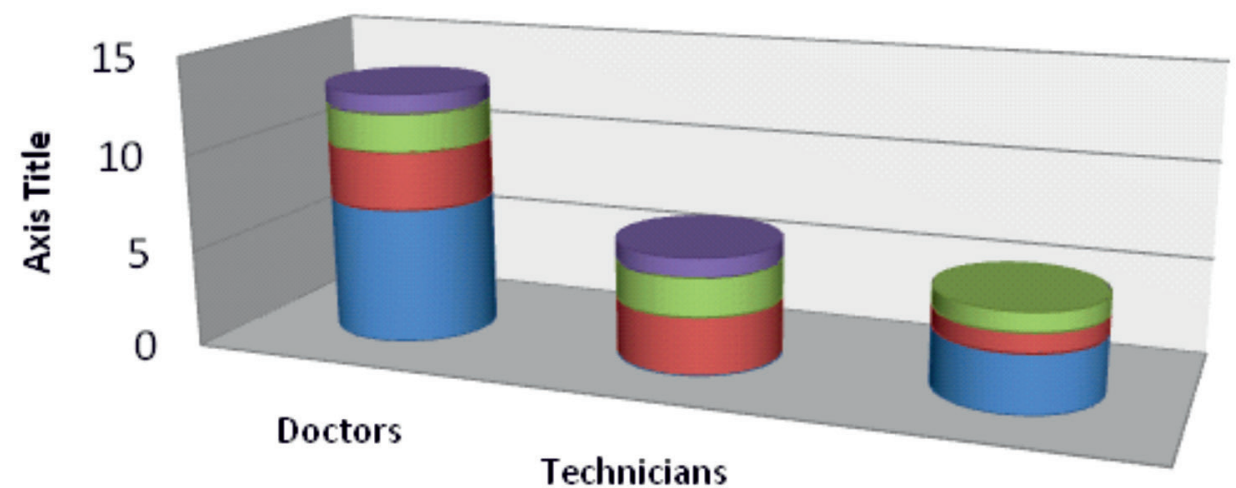

Nurses

\begin{tabular}{|l|c|c|c|}
\cline { 2 - 4 } \multicolumn{1}{c|}{} & Doctors & Technicians & Nurses \\
\hline$\square$ VRE & 1 & 1 & 0 \\
\hline$\square$ MRSA & 2 & 2 & 1 \\
\hline$\square$ MDR Pseudomonas & 3 & 3 & 1 \\
\hline$\square$ MDR Acinetobacter & 7 & 0 & 3 \\
\hline
\end{tabular}

Fig. 1: Distribution of MDR bacteria amongst HCW 
(10.6\%).A higher incidence of contamination of doctors compared with the incidence of contamination of nurses and technicians, is especially concerning, because physicians typically see more patients and have lower rates of hand hygiene than other HCWs ${ }^{[13]}$.

Our analysis showed more frequent MDR colonization amongst the male staff. This finding is similar to that reported in previous studies ${ }^{[14,15]}$ ). Also significant colonization was observed in HCW who had sufferd from a wound or ulcer on an exposed part of the body in last six months, corroborated by a previous study $\left.{ }^{[14]}\right]$. This can be adquetely explained by the fact that break in integrity of skin makes it easier for organisms to gain access to the body.

Also a significant association was found between MDR colonization and close contact with ICU patients which is different from that reported by Petres et $\mathrm{al}^{[15,16]}$ For the purpose of the study close contact with patient was defined as changing dressing, or assisting mobility or carrying out an invasive procedure. These procedures make the $\mathrm{HCW}$ at risk of acquiring bacterial flora because of contact with contaminated wound, pus, blood or other body fluids. Other factors did not show significant association with colonization of HCW.

We did not collect patient-specific information, so were unable to assess whether the ICU patients were actually the cause of this contamination of hands of $\mathrm{HCW}$.However, as is evident from the demographic data collected, most HCW have been working in close contact with ICU patients for a considerable period of time and many of them had one or more known risk factor for acquiring bacteria flora. Therefore, they have more chances of having acquired the bacterial pathogens from patients either through direct contact or indirectly from contaminated environment than from any other source. Nevertheless, for confirmation of whether the same isolate has been transferred between HCW and patients, molecular characterization is needed and this remains a limitation of our study.

\section{Conclusion}

Health Care Associated Infections especially in the vulnerable ICU patient population can be linked to the bacterial flora colonizing the HCWs. This flora is acquired during routine patient care activity either due to direct patient contactor indirectly through contaminated environment or surfaces. This contaminating flora is more likely to be MDR in ICU patients due to risk factors associated with these patients such a prolonged use multiple antibiotics. Determining the prevalence of MDR bacteria on the hands of HCW and the associated risk factors can help the hospital in preparing an effective strategy for curbing the menace of Health care associated infections. Further, investigation of the isolates from nasal swabs and or hands of healthcare workers and simultaneously form ICU patients by molecular studies and genotyping is desirable, in order to establish the authenticity, identity and inter- relationship of the isolated organisms, and prove their roles in infection causation.

\section{Acknowledgements}

The authors would like to express their sincere thanks to senior technician Vijay laxmi Bahuguna and Infection control nurse Sonia for their valuable help during the course if study.

\section{Reference}

1. Morgan DJ, Liang SY, Smith CL, Johnson JK, Harris AD. et al. Frequent Multidrug-Resistant Acinetobacter baumannii contamination of Gloves, Gowns, and Hands of Healthcare. Infect Control Hosp Epidemiol . 2010 July; 31(7): 716-721.

2. Gaynes R, Edwards JR. National Nosocomial Infections Surveillance System. Overview of nosocomial infections caused by gram-negative bacilli. Clin Infect Dis 2005;41: 848-854.

3. Harbarth S, Fankhauser C, Schrenzel J, et al. Universal screening for methicillin-resistant Staphylococcus aureus at hospital admission and nosocomial infection in surgical patients. JAMA 2008; 299:1149-57.

4. Barbolla RE, Centron D, Maimone S, et al. Molecular epidemiology of Acinetobacter baumannii spread in an adult intensive care unit under an endemic setting. Am J Infect Control 2008;36: 444-452.

5. Olsen R, Lynch P, Coyle M, Cummings J, Bokete T, Stamm $\mathrm{W}$. Examination gloves as barriers to hand contamination in clinical practice. JAMA 1993; 270:350-353.

6. Sassmannshausen R, Deurenberg RH, Kock R, et al. MRSA Prevelance and associated risk factors among Health care workers in non outbreak situations in the Dutch - German EUREGIO. Frontiers in Microbiology. 2016;7:1273

7. Clinical and laboratory Standards Institute. Performance Standards for antimicrobial testing; fifteenth informational supplement M10-S14, wayne, PA, CLSI. 2016

8. Go ES, Urbans C, Burns J, Kreiswirth B, Eisner W, Mariano N, et al. Clinical and molecular epidemiology of acinetobacter infections sensitive only to polymyxin B and sulbactum. Lancet. 1994; 344:1329-32.

9. Malini J, Shruti A, Padmavathy M, Umapathy B.L,NAvaneeth B.V, et al. Methicillin resistant Staphylococcus carriage among the health workers in a tertiary care hospital. JCDR 2012 June;6:791-93. 
10. Mathanraj S, Sujatha S, Sivasangeetha K, Parija SC. Screening for methicillin resistant Staphylococcus aureus carriers among the patients and health care workers of a tertiary care hospital in south Indian J Med Microbiology 2009;27(1):62-64.

11. Sood S, Malhotra M, Das BK, Kapil A. Enterococcal infections and antimicrobial resiatnace. Indian J Med Res 2008;128:111-21.

12. Khodavaisy S, Nabili M, Davari B, Vahedui M. Evaluation of bacterial anti-fungal contamination in health care worker's hands and ring $\mathrm{s}$ in the intensive care unit. J prev Med Hyg 2011;52:215-218.
13. Peleg AY, Seifert H, Paterson DL. Acinetobacter baumannii: emergence of a successful pathogen. Clin Microbiol Rev 2008; 21:538-582.

14. Kamble R. Acinetobacter species in health care setting: Clinical significance and antimicrobial sensitivity. Int J Curr Microbiolo App Sci 2015;4(4):861-869.

15. Pters C, Dulon M, Kleinmuller O, Nienhaus A, Scblon A. MRSA prevelance and risk factors among health personnel and residents in nursing homes in Hanburg , Germany- a cross sectional study. PLOS ONE 2017;12(1) :e0169425.

16. Robicsek A, Beaumont JL, Paule SM, et al. Universal surveillance for methicillin-resistant Staphylocloccus aureus in 3 affiliated hospitals. Ann Intern Med 2008;148:409-418.

*Corresponding author:

Dr. Iva Chandola, Assistant Professor, Dept of Microbiology, Shri Guru Ram Rai Institute of Medical and Health Sciences, Dehradun, India

Email: chandolaiva@gmail.com

Date of Submission : 19.04.2017

Date of Acceptance : 28.06.2017

Financial or other Competing Interests: None.

Date of Publication : 26.10.2017 